\title{
INJECTIVE DIMENSION OF SOME DIVISIBLE MODULES OVER A VALUATION DOMAIN
}

\author{
SILVANA BAZZONI \\ (Communicated by William C. Waterhouse)
}

\begin{abstract}
Let $R$ be a valuation domain of global dimension $n+1$. Given an infinite direct product of injective envelopes of (torsion) cyclic modules, let $D_{n-k}$ be the submodule consisting of the elements having support of cardinality less than $\aleph_{n-k}$.

We prove that the injective dimension of $D_{n-k}$ is at most $k$ and, using $\diamond$-axiom, we prove that $D_{n-2}$ has injective dimension exactly 2 .
\end{abstract}

Introduction. Given a valuation domain $R$ it is well known that, if every ideal of $R$ is countably generated, then every divisible $R$-module is either injective or of injective dimension 1. (See [FS, Chapter 6].) Very little seems to be known if $R$ admits ideals of uncountable type or, equivalently if global dimension $R \geq 3$.

Here we address this question for $R$-modules of a particular type. Let global dimension $R=n+1(\geq 3)$; given an infinite direct product of injective envelopes of cyclics $R / L_{\alpha}\left(L_{\alpha}\right.$ archimedean ideals) consider the submodule $D_{n-k}(0 \leq k \leq n)$ of the product consisting of all the elements having support of cardinality $<\aleph_{n-k}$. We prove that the injective dimension of $D_{n-k}$ is at most $k$, while $D_{n-1}$ has injective dimension exactly 1 .

The case $k=2$ is more difficult. Using the combinatorial principle known as $\diamond$-axiom we prove that $D_{n-2}$ has injective dimension 2 .

If global dimension $R=3$, the module $D_{n-2}$ is a direct sum of injectives and we do not know if it is consistent with ZFC to assume that its injective dimension is 1 .

We are indebted to Professor L. Fuchs for proposing to us the question discussed in this paper and for suggesting to us the use of $\diamond$-axiom for its solution.

1. The modules $D_{n-k}$. $R$ will always denote a valuation domain, $P$ its maximal ideal and $Q$ its quotient field.

If $J$ is an ideal of $R$, gen $J$ will denote the least cardinality of generating systems for $J$ and we will say that $J$ is $\kappa$-generated (for a cardinal $\kappa$ ) in case gen $J \leq \kappa$. $\left(\aleph_{-1}\right.$ means finitely generated.)

If $M$ is an $R$-module and $J$ and ideal of $R, M[J]$ will denote the submodule of $M$ consisting of the elements annihilated by $J$. Throughout we will use well-known results about the projective dimension of modules over $R$ (see [O]).

Let global dimension $R=n+1$. Baer's criterion says that an $R$-module $M$ is injective if and only if $\operatorname{Ext}^{1}(R / J, M)=0$ for every $\aleph_{n-1}$-generated ideal $J$.

The object of the present paper is to investigate the following problem.

Received by the editors September 19, 1986 and, in revised form, March 13, 1987.

1980 Mathematics Subject Classification (1985 Revision). Primary 13C11. 
Problem 1. Suppose $M$ is an $R$-module satisfying $\operatorname{Ext}^{1}(R / J, M)=0$ for every $\aleph_{m}$-generated ideal $J$, for some $m \in N$. What can be said about the injective dimension of $M$ ?

The first result we can establish is the following.

Proposition 1. Let global dimension $R=n+1$ and $M$ an $R$-module such

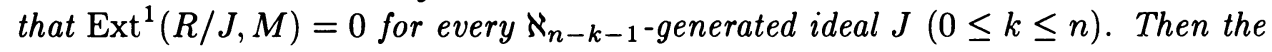
injective dimension of $M$ is at most $k$.

Proof (The case $k=0$ is just Baer's criterion). We first prove that $(*)$ $\operatorname{Ext}^{1}(N, M)=0$ for every $R$-module $N$ of projective dimension at most $n-k+1$.

In fact, by Corollary 4 in [BF], we have $N=\bigcup_{\alpha<\tau} N_{\alpha}$ where $\left\{N_{\alpha}\right\}_{\alpha<\tau}$ is a smooth chain of submodules of $N$ such that $N_{\alpha+1} / N_{\alpha} \approx R / J$ with $J \aleph_{n-k-1^{-}}$ generated. A simple application of Lemma 2.1, Chapter IV [FS] yields (*). Using induction on $m$ we can now prove $\operatorname{Ext}^{m+1}(N, M)=0$ for every $R$-module $N$ of projective dimension at most $n-k+1+m$.

The case $m=0$ is just $(*)$.

Let $N$ be of projective dimension at most $n-k+1+m$ and consider an exact sequence: (1) $0 \rightarrow N_{1} \rightarrow F \rightarrow N \rightarrow 0$ where $F$ is a free module; then projective dimension $N_{1} \leq n-k+m$ and by induction $\operatorname{Ext}^{m}(N, M)=0$. But the exact sequence $(1)$ yields $\operatorname{Ext}^{m}\left(N_{1}, M\right) \approx \operatorname{Ext}^{m+1}(N, M)$.

This natural question now arises:

QUESTION 1. Let $R$ and $M$ be as in Proposition 1. Assume there exists an ideal $J$ such that gen $J=\aleph_{n-k}$ and $\operatorname{Ext}^{1}(R / J, M) \neq 0$. Can we prove that the injective dimension of $M$ is exactly $k$ ?

The hypothesis $\operatorname{Ext}^{1}(R / J, M)=0$ for every $\aleph_{n-k-1}$-generated ideal $J$, implies $\operatorname{Ext}^{1}(R / J, M)=0$ for every finitely generated ideal $J$ (since $k \geq 0$ ) and this is equivalent to saying that $M$ is divisible. So from now on we will assume $M$ is divisible and moreover we will concentrate on a special class of divisible modules.

We now set down some notations which will be frequently used in the sequel.

Let $\left\{L_{\alpha}\right\}_{\alpha \in \Lambda}$ be a family of archimedean ideals of $R$ (i.e. $r L_{\alpha} \supsetneqq L_{\alpha}$, for every nonunit $r \in R$ ) and let $I_{\alpha}$ be the injective envelope of $R / L_{\alpha}, \alpha \in \Lambda$. (Note that $I_{\alpha} \geq Q / L_{\alpha}$.) For $n \in N$ and $0 \leq k \leq n$ denote by $D_{n-k}$ the submodule of $\prod_{\alpha \in \Lambda} I_{\alpha}$ consisting of all the elements whose supports have cardinality less than $\aleph_{n-k}$. We obviously assume $\Lambda$ is big enough, namely $|\Lambda| \geq \aleph_{n}$. The aim of the following two propositions is to establish that the module $D_{n-k}$ satisfies the requirements mentioned in Question 1.

For every ideal $J$ of $R$ we need to consider a filtration. Assume gen $J=\aleph_{k}$; we construct, by transfinite induction, a smooth chain $\left\{J_{\lambda}\right\}_{\lambda<\aleph_{k}}$ of ideals in $J$ as follows.

$J_{1}$ is generated by an element $j_{1}$ in $J$.

If $\lambda$ is a limit ordinal $\left(\lambda<\aleph_{k}\right), J_{\lambda}=\bigcup_{\nu<\lambda} J_{\nu}$.

If $\nu$ is nonlimit, $\nu<\aleph_{k}, J_{\nu}$ is generated by an element $j_{\nu}$ in $J$ satisfying: (i) if $\nu=\mu+1, \mu$ nonlimit, $j_{\nu}$ is an element not in $J_{\mu}$. (ii) If $\nu=\lambda+1, \lambda$ limit, $j_{\nu}$ is an element not in $J_{\lambda}\left(=\bigcup_{\mu<\lambda} J_{\mu}\right)$ with $\left\langle j_{\nu}\right\rangle / J_{\lambda}$ not isomorphic to $R / P$ in case $P$ is not principal.

Next we want $J=\bigcup_{\lambda<\kappa_{k}} J_{\lambda}$. It is easy to see that such a filtration always exists for every ideal $J$. We will call it a regular filtration. The point to be checked is 
the construction of the element $j_{\nu}, \nu=\lambda+1, \lambda$ limit; satisfying condition (ii). Consider $a \in J \backslash J_{\lambda}$; assume $P$ is not principal and $\langle a\rangle / J_{\lambda}$ isomorphic to $R / P$. This means $R / a^{-1} J_{\lambda}$ isomorphic to $R / P$ or, equivalently, $a^{-1} J_{\lambda}=P$. Let $b$ be an element in $J \backslash\langle a\rangle$; there is $r \in P$ such that $r b=a$ and thus $\langle b\rangle / J_{\lambda}$ cannot be isomorphic to $R / P$ since $b^{-1} J_{\lambda}=r a^{-1} J_{\lambda}=r P$ which is strictly contained in $P$. Put then $b=j_{\nu}$.

\section{Proposition 2. $\operatorname{Ext}^{1}\left(R / J, D_{n-k}\right)=0$ for every ideal $J$ such that gen $J \neq$ $\aleph_{n-k}$.}

PROOF. We prove that if $J$ is as stated and $f: J \rightarrow D_{n-k}$ is a homomorphism, then $f$ is extendable to $R$. Let $\left\{J_{\lambda}\right\}_{\lambda<\operatorname{gen} J}$ be a regular filtration for $J$. Define the support of $f$ as the union of the supports of the elements $f\left(j_{\nu}\right)$, for $\nu$ nonlimit, $\nu<\operatorname{gen} J$.

Case 1. gen $J \supsetneqq \aleph_{n-k}$. It is clear that in this case $|\operatorname{supp} f| \supsetneqq \aleph_{n-k}$; thus $f(J) \leq \prod_{\alpha \in \Lambda_{1}} I_{\alpha}$ where $\Lambda_{1}=\operatorname{supp} f$, and $f$ is extendable to $R$.

Case 2. gen $J \supsetneqq \aleph_{n-k}$. As in the first case it suffices to prove that $|\operatorname{supp} f| \supsetneqq$ $\aleph_{n-k}$. By way of contradiction suppose $|\operatorname{supp} f| \geq \aleph_{n-k}$ and choose $J_{0}$ in $J, J_{0}$ $\aleph_{n-k}$-generated with $|\operatorname{supp} f|_{J_{0}} \mid=\aleph_{n-k} .\left(\left.f\right|_{J_{0}}\right.$ denotes the restriction of $f$ to $\left.J_{0}.\right)$ Take $j \in J \backslash J_{0}, j R \supsetneqq J_{0}$; hence $\left.\operatorname{supp} f(j) \supseteq \operatorname{supp} f\right|_{J_{0}}$ which is absurd since $\operatorname{supp} f(j)$ has to be of cardinality less than $\aleph_{n-k}$.

PROPOSITION 3. $\operatorname{Ext}^{1}\left(R / J, D_{n-k}\right) \neq 0$ for every ideal $J$ with gen $J=\aleph_{n-k}$. Moreover given an increasing chain $\left\{X_{\nu}: \nu\right.$ nonlimit, $\left.\nu<\aleph_{n-k}\right\}$ of subsets of $\Lambda$ each one of cardinality less than $\aleph_{n-k}$, and a regular filtration $\left\{J_{\lambda}\right\}_{\lambda<\aleph_{n-k}}$, there is a homomorphism $f: J \rightarrow D_{n-k}$ such that $\left.\operatorname{supp} f\right|_{J_{\nu}}=X_{\nu}$, for every nonlimit $\nu<\aleph_{n-k}$.

PROOF. It is enough to prove the second part since, if we choose the chain $\left\{X_{\nu}\right\}$ satisfying $\left|\bigcup_{\nu} X_{\nu}\right|=\aleph_{n-k}$, then the stated homomorphism $f$ is not extendable to $R$.

Let $\mu, \nu$ denote nonlimit ordinals, $\mu<\nu<\aleph_{n-k}$. We have $r_{\mu}^{\nu} j_{\nu}=j_{\mu}$ for $r_{\mu}^{\nu}$ non units in $R$ and $j_{\mu}, j_{\nu}$ the generators of the corresponding elements $J_{\mu}, J_{\nu}$ in the regular filtration.

By transfinite induction define elements $d_{\nu}$ in $D_{n-k}$ such that (i) $r_{\mu}^{\nu} d_{\nu}=d_{\mu}$, (ii) supp $d_{\nu}=X_{\nu}$. Then $f\left(j_{\nu}\right)=d_{\nu}$ will yield the required homomorphism. Let $d_{1}$ be any element of $D_{n-k}$ with support $X_{1}$. Suppose $d_{\mu}$ has been chosen for every $\mu<\nu$. Define $d_{\nu}$ as follows.

Case 1. $\nu=\mu+1, \mu$ nonlimit. If $\alpha \in X_{\mu}$, let $d_{\nu}(\alpha)$ be a nonzero element in $I_{\alpha}$ such that $r_{\mu}^{\nu} d_{\nu}(\alpha)=d_{\mu}(\alpha)$. If $\alpha \in X_{\nu} \backslash X_{\mu}$ let $d_{\nu}(\alpha)$ be $q+L_{\alpha}$ with $q$ in $\left(r_{\mu}^{\nu}\right)^{-1} L_{\alpha} \backslash L_{\alpha}$; there exists such a $q$ by the hypothesis $L_{\alpha}$ archimedean. Obviously $d_{\nu}(\alpha)=0$ if $\alpha \notin X_{\nu}$.

Case 2. $\nu=\lambda+1, \lambda$ limit. Let $\Lambda_{0}=\bigcup_{\mu}\left\{X_{\mu}: \mu<\nu\right\}$. Clearly $\left|\Lambda_{0}\right| \varsubsetneqq \aleph_{n-k}$ and the elements $d_{\mu}(\mu<\nu)$ already constructed lie in $\prod_{\alpha \in \Lambda_{0}} I_{\alpha}=I_{0}$. The system of equations $r_{\mu}^{\nu} x=d_{\mu}$ is finitely solvable in $I_{0}$ and hence it has a solution $d$ in $I_{0}$; put $d_{\nu}(\alpha)=d(\alpha)$ for $\alpha \in \Lambda_{0}$. For $\alpha \in X_{\nu} \backslash \Lambda_{0}$ let $d_{\nu}(\alpha)=q+L_{\alpha}$ with $q$ an element of $\bigcap_{\mu}\left\{\left(r_{\mu}^{\nu}\right)^{-1} L_{\alpha} \backslash L_{\alpha}, \mu<\nu, \mu\right.$ nonlimit $\}$. The intersection is not empty by property (ii) of a regular filtration. 
Let $d_{\nu}(\alpha)=0$ for $\alpha \notin X_{\nu}$. Obviously the family $\left\{d_{\nu}: \nu<\aleph_{n-k}, \nu\right.$ nonlimit $\}$ satisfies (i) and (ii) above and thus the conclusion follows.

2. The injective dimension of $D_{n-2}$. In this section we inquire about the injective dimension of the modules $D_{n-k}$.

Assume global dimension $R=n+1$. We have:

FACT 1. Injective dimension $D_{n-k} \leq k$.

Obvious from Propositions 1 and 2. Thus, in particular, injective dimension $D_{n}=0$.

FACT 2. Injective dimension $D_{n-1}=1$.

In fact $D_{n-1}$ is not injective by Proposition 3. Thus the interesting case is for $n \geq 2$, hence we will assume global dimension $R \geq 3$.

Now, by Fact 1 and Proposition 3, we infer that the injective dimension of $D_{n-2}$ is 1 or 2 .

The exact value does not seem decidable in ZFC. So from now on we will concentrate on $D_{n-2}$. First of all, notice that, if $\Lambda_{1}$ is a subset of $\Lambda$ of cardinality $\aleph_{n-2}$, then the elements of $D_{n-2}$ with supports in $\Lambda_{1}$ form a direct summand of $D_{n-2}$. Thus if we want to prove that injective dimension $D_{n-2}=2$ we can assume $|\Lambda|=\aleph_{n-2}$. Consider the exact sequence

$$
0 \rightarrow D_{n-2} \rightarrow I \rightarrow C \rightarrow 0 \text { where } I=\prod_{\alpha \in \Lambda} I_{\alpha},|\Lambda|=\aleph_{n-2} .
$$

By the previous remark, the injective dimension of $C$ is 0 or 1 and clearly $C$ is injective if and only if every homomorphism from every $J$ to $C$ has a lifting to $I$.

LEMMA 1. Let $J$ be $\aleph_{n-2}$-generated and $\left\{J_{\lambda}\right\}_{\lambda<\text { gen } J}$ a regular filtration for $J$. Then

(i) $\operatorname{Ext}^{1}\left(J, D_{n-2}\right)=0$.

(ii) $\operatorname{Hom}(J, I) \rightarrow \operatorname{Hom}(J, C) \rightarrow 0$ is exact.

(iii) Given a family $\left\{y_{\nu}: \nu\right.$ nonlimit, $\nu<$ gen $\left.J\right\}$ of elements of $I$ with

(a) $j_{\mu}\left(y_{\nu}-y_{\mu}\right) \in D_{n-2}$ for every $\mu<\nu<\operatorname{gen} J$ ( $\mu$ nonlimit), there exists an element $y \in I$ such that

(b) $j_{\nu}\left(y-y_{\nu}\right) \in D_{n-2}$ for every $\nu<\operatorname{gen} J$.

PrOOF. (i) Write $J=\bigcup_{\lambda<\text { gen } J} J_{\lambda}$. Then $J_{\lambda+1} / J_{\lambda}$ is isomorphic to $R / J^{\prime}$ with gen $J^{\prime} \supsetneqq \aleph_{n-2}$. Thus, by Proposition $2, \operatorname{Ext}^{1}\left(J_{\lambda+1} / J_{\lambda}, D_{n-2}\right)=0$ and by Lemma 2.1, Chapter 2 of [FS] the conclusion follows.

(ii) Obvious from (i) and the exact sequence (1).

(iii) is just a reformulation of (ii) since condition (a) guarantees that $f\left(j_{\nu}\right)=$ $j_{\nu}\left(y_{\nu}+D_{n-2}\right)$ is a homomorphism $f: J \rightarrow C$ and $f$ lifts to $I$ exactly if there exists $y \in I$ satisfying condition (b).

The following technical lemma is crucial.

LEMMA 2. Let $J$ be an ideal, gen $J=\aleph_{n-2}$ and $\left\{J_{\lambda}\right\}_{\lambda<\aleph_{n-2}}$ a regular filtration for J. Let $\left\{y_{\nu}: \nu\right.$ nonlimit, $\left.\nu<\aleph_{n-2}\right\}$ be a family of elements of $I$ satisfying condition (a) of Lemma 1(iii). Given a family $\left\{H_{\nu}: \nu\right.$ nonlimit, $\left.\nu<\aleph_{n-2}\right\}$ of $\aleph_{n-2}$ subsets of $\Lambda$ of cardinality less than $\aleph_{n-2}$, there exist $y \in I$ and a subset $A$ of $\Lambda, A=\left\{\alpha_{\nu}: \nu<\aleph_{n-2}, \nu\right.$ nonlimit $\}$ such that

(i) $|A|=\aleph_{n-2}$. 
(ii) For every $\nu, \alpha_{\nu} \notin H_{\nu}$; $\operatorname{supp} j_{\nu}\left(y-y_{\nu}\right)$ has cardinality less than $\aleph_{n-2}$ and contains $\alpha_{\nu}$.

PROOF. By Lemma 1(iii) there exists $y_{0} \in I$ satisfying (b), namely $j_{\nu}\left(y_{0}-y_{\nu}\right) \in$ $D_{n-2}$, for every $\nu$ and clearly any other element of $I$ satisfying (b) is in the coset $\left(y_{0}+D_{n-2}\right)+C[J]$. We want to find an element $z \in I$, with $z+D_{n-2} \in C[J]$, in order that $y_{0}+z$ will be the required element. Let $\mu, \nu$ be nonlimit ordinals $<\aleph_{n-2}$. Put $G_{\nu}=\operatorname{supp} j_{\nu}\left(y_{0}-y_{\nu}\right)$ and define $A$ inductively as follows: $\alpha_{1}$ is an element in $\Lambda \backslash\left(G_{1} \cup H_{1}\right)$ and if $\alpha_{\mu}$ has been chosen for every $\mu<\nu$, let $\alpha_{\nu}$ be an element in $\Lambda \backslash\left(G_{\nu} \cup H_{\nu} \cup\left\{\alpha_{\mu}: \mu<\nu\right\}\right)$. Obviously $|A|=\aleph_{n-2}$. For every $\nu$, let $F_{\nu}=\left\{\alpha_{\mu}: \mu \leq \nu\right\}$. We can apply Proposition 3 to the family $\left\{F_{\nu}: \nu<\aleph_{n-2}\right\}$ to get an element $z \in I$ with $\operatorname{supp} j_{\nu} z=F_{\nu}$, for every $\nu$. Take $y=y_{0}+z$. Then for every $\nu, j_{\nu}\left(y-y_{\nu}\right)=j_{\nu} z+j_{\nu}\left(y_{0}-y_{\nu}\right)$. Consequently, supp $j_{\nu}\left(y-y_{\nu}\right) \subseteq F_{\nu} \cup G_{\nu}$ and since $\alpha_{\nu} \in F_{\nu} \backslash G_{\nu}$, we get $\alpha_{\nu} \in \operatorname{supp} j_{\nu}\left(y-y_{\nu}\right)$.

Up to now, we know that ever homomorphism from an $\aleph_{n-2}$-generated ideal $J$ into $C$ lifts to $I$ (Lemma 1). But, since global dimension $R=n+1$, there are ideals $J$ with gen $J=\aleph_{n-1}$, and to be able to find homomorphisms from such ideals to $C$ not liftable to $I$, we assume the axiom $\diamond_{\kappa_{n-1}}$ (due to Jensen) which we are going to use in the following form (see $[\mathbf{E}]$ ).

Let $X$ be a set $|X| \leq \aleph_{n-1}, X=\bigcup_{\lambda<\aleph_{n-1}} X_{\lambda}$ (smooth filtration), there is a family of functions ( $\diamond$-sequence), $\left\{h_{\lambda}: \lambda \rightarrow X_{\lambda} \mid \lambda<\aleph_{n-1}\right\}$ such that, for any function $h: \aleph_{n-1} \rightarrow X$, the set $\left\{\lambda<\aleph_{n-1}|h|_{\lambda}=h_{\lambda}\right\}$ is stationary.

THEOREM 1. Assume $\diamond_{\aleph_{n-1}}+\left(2^{\aleph_{n-3}} \leq \aleph_{n-1}\right.$, if $\left.n \geq 3\right)$. Then the injective dimension of $D_{n-2}$ is 2 .

PROOF. We show that for every ideal $J$ with gen $J=\aleph_{n-1}$, there exists a homomorphism $f: J \rightarrow C$ not liftable to $I$; this will imply injective dimension $D_{n-2}=2$.

Let $\left\{J_{\lambda}\right\}_{\lambda<\aleph_{n-1}}$ be a regular filtration for $J$. We define inductively the restrictions $\left.f\right|_{J_{\lambda}}$ of $f$ to $J_{\lambda}$. They have to be multiplications by elements $y_{\lambda}+D_{n-2}$, $y_{\lambda} \in I$, since gen $J_{\lambda}<\aleph_{n-1}$ for every $\lambda<\aleph_{n-1}$ (apply Lemma 1). Obviously the elements $y_{\lambda}$ satisfy

$$
j_{\mu}\left(y_{\lambda}-y_{\mu}\right) \in D_{n-2} \quad \text { for every } \mu<\lambda<\aleph_{n-1}, \mu \text { nonlimit. }
$$

Let $X$ be the set of all subsets of $\Lambda$ of cardinality less than $\aleph_{n-2}$. Then $|X|=$ $2^{\aleph_{n-3}} \leq \aleph_{n-1}$ by assumption (in case $n=2,|X|=\aleph_{0}$ without any hypothesis). Write $X=\bigcup_{\lambda<\kappa_{n-1}} X_{\lambda}$ (smooth filtration) and consider a $\diamond_{\aleph_{n-1}}$-sequence $h_{\lambda}: \lambda \rightarrow$ $X_{\lambda}, \lambda<\aleph_{n-1}$. We define, by induction, a family $\left\{y_{\lambda} \in I: \lambda<\aleph_{n-1}\right\}$ satisfying $(*)$.

(i) $\lambda=1, y_{1}$ is any element in $I$.

(ii) $\lambda$ limit or $\lambda=\rho+1$ for $\rho$ limit such that $|\rho| \supsetneqq \aleph_{n-2}$.

Consider the already constructed set $\left\{y_{\mu}: \mu\right.$ nonlimit, $\left.\mu<\lambda\right\}$ satisfying $(*)$. Let $y_{\lambda}$ be an element satisfying condition (b) of Lemma 1 (iii).

(iii) $\lambda=\nu+1, \nu$ nonlimit. Let $y_{\lambda}$ be such that $j_{\nu}\left(y_{\lambda}-y_{\nu}\right) \in D_{n-2}$.

(iv) $\lambda=\rho+1, \rho$ limit, $|\rho|=\aleph_{n-2}$. 
Consider the function $h_{\rho}: \rho \rightarrow X_{\rho}$ and put $h_{\rho}(\mu)=H_{\mu}$ for every $\mu<\rho$ $\mu$ nonlimit. The family $\left\{H_{\mu}: \mu<\rho\right\}$ satisfies the hypothesis of Lemma 2 , therefore we can find an element $y \in I$ such that, if $S_{\mu}=\operatorname{supp} j_{\mu}\left(y-y_{\mu}\right), S_{\mu}$ contains an element $\alpha_{\mu} \in \Lambda \backslash H_{\mu}$ and $\left|\left\{\alpha_{\mu}: \mu<\rho\right\}\right|=\aleph_{n-2}$. Let $y_{\lambda}$ be such a $y$. It is clear that the set $\left\{y_{\lambda}: \lambda<\aleph_{n-1}\right\}$ constructed above satisfies $(*)$ and thus $f=\bigcup_{\lambda}\left\{\left.f\right|_{J_{\lambda}}: \lambda<\aleph_{n-1}\right\}$ gives rise to a homomorphism $f: J \rightarrow C$.

ClAIM. $f$ is not liftable to $I$.

Suppose the contrary. This means there is a family $\left\{d_{\lambda}: d_{\lambda} \in D_{n-2}, \lambda<\aleph_{n-1}\right\}$ satisfying $(* *) j_{\mu}\left(y_{\lambda}+d_{\lambda}-\left(y_{\mu}+d_{\mu}\right)\right)=0$ for every $\mu<\lambda<\aleph_{n-1}, \mu$ nonlimit. Consider the function $h: \aleph_{n-1} \rightarrow X, h(\lambda)=\operatorname{supp} d_{\lambda}=F_{\lambda}$, for every $\lambda<\aleph_{n-1}$. By $\diamond_{\aleph_{n-1}}$, there is a limit ordinal $\rho_{0}$ with $\left|\rho_{0}\right|=\aleph_{n-2}$ (the set of such $\rho_{0}$ 's is stationary), such that $h\left(\rho_{0}\right)=\left.h\right|_{\rho_{0}}$, i.e. $H_{\mu}=F_{\mu}$ for every $\mu<\rho_{0}, \mu$ nonlimit.

Consider the element $y_{\rho_{0}+1}$ constructed in (iv). We have $j_{\mu}\left(y_{\rho_{0}+1}-y_{\mu}\right)=$ $-j_{\mu}\left(d_{\rho_{0}+1}-d_{\mu}\right)$, for every $\mu<\rho_{0}, \mu$ nonlimit; hence, $S_{\mu}=\operatorname{supp} j_{\mu}\left(y_{\rho_{0}+1}-y_{\mu}\right) \subseteq$ $F_{\rho_{0}+1} \cup F_{\mu}$. but $\alpha_{\mu} \in S_{\mu} \backslash F_{\mu}$, implies $\alpha_{\mu} \in F_{\rho_{0}+1}$, for every $\mu<\rho_{0}$, contradicting $\left|F_{\rho_{0}+1}\right| \supsetneqq \aleph_{n-2}$.

REMARK. Let global dimension $R=3 . D_{n-2}$ is just $\bigoplus_{n<\omega} I_{n}$ and Theorem 1 says that $\diamond_{K_{1}}$ implies injective dimension $\bigoplus_{n<\omega} I_{n}=2$.

We end with this natural question.

Is there an axiom which implies that injective dimension $\bigoplus_{n<\omega} I_{n}=1$ ?

\section{REFERENCES}

[BF] S. Bazzoni and L. Fuchs, On modules of finite projective dimension over valuation domains, Proc. Conf. on Abelian Groups and Modules (Udine, 1984), Springer, Wien and New York, 1984, pp. 361-371.

[E] P. Eklof, Set theoretic methods in homological algebra and abelian groups, Presses Univ. Montréal, Montréal, 1980.

[FS] L. Fuchs and L. Salce, Modules over valuation domains, Dekker, New York, 1985.

[O] B. Osofsky, Glboal dimension over valuation rings, Trans. Amer. Math. Soc. 1.27 (1967), 136149.

Dipartimento di Matematica, Università di Padova, 35100 Padova, Italy 